\title{
A New Models for Emergency Evacuation under the Disaster Condition
}

\author{
Luliang TANG ${ }^{*}$, Xue YANG ${ }^{*}$, Fangzhen HUANG, Hong Xu, Qingquan LI
}

\begin{abstract}
State Key Laboratory of Information Engineering in Surveying, Mapping and Remote Sensing, Wuhan University, Wuhan, 430079, P. R. China
\end{abstract}

KEY WORDS: Emergency evacuation, Multi-level emergency evacuation model, Spatial point matching, The K routs, Car assignment, Emergency evacuation platform

\begin{abstract}
:
People must face many disasters every day, which cause losses in property and human lives. It is significant how to establish the emergency evacuation model and for high density population in large areas according to the actual situation of their emergency evacuation. This paper put forward a Multi-levels Emergency Evacuation Model (MEE) based on the collective evacuation for high density of population in large areas under the earthquake condition in China, which firstly find the best ways from the multi-gathering points(Origins) in the disaster area to multi-settling points(Destinations )in the safe area based on the road impedance such as the traffic, road speed limit and pavement damage to weight the evacuation ability of road network, and secondly determine the evacuation auto numbers on the every routes which is at the target to deliver all the evacuating people from the Origins to Destinations in the shortest time. The experiment area is Dezhou city in Shandong province of China, and an emergency evacuation platform was put up according to the multilevel emergency evacuation model using Google map API and C\# as development platform, and the result show us that MEE is promised to be a new methods for emergency evacuation.
\end{abstract}

\section{INTRODUCTION}

The disasters were recurrent around the world during the last several years. Taking some cases for example, on May 12, 2008, the grievous earthquake struck Wenchuan County, Sichuan province in southwestern China that has taken 70,000 people away, made 370,000 wounded and more than 10,000 disappeared or so. The day on April 14, 2010, the quake which hit 7.1 on the Richter scale happened in Yushu county, Qinghai province with 2, 220 people dead and 70 missing. The more terrible is the 9.0 magnitude earthquake in the eastern waters of Honshu island that resulted in 13498 deaths and 14734 disappearances on March 11, this year. They are not unique, but have their counterparts. On October 23, this year, 481 were deceased in eastern Van province of Turkey under the quake measuring 7.2. The awesome figures by quakes show that the recurrent earthquake disasters have a serious influence on social stability, development and public safety. So, coping with emergencies to safeguard the public lives and properties has being the cardinal issue of researchers all over the world. Model for emergency evacuation has to start with road network, with affecting the efficiency, and selecting evacuation path which transfers the stricken persons from hazardous area to secure area is the principal problem of constructing the model for emergency evacuation. From 1960s on, the researchers have proposed many emergency evacuation models for kinds of disasters: In the $1970 \mathrm{~s}$, Lewis(1985) proposed transportation planning for hurricane evacuations. In the next followed decade, the Virginia State of America advanced NETVACI (Antoine G et al, 1998). Cova (2003) put forward a network optimal flow model for lane based evacuation routing. Jernigan and Post.etc (2000)proposed macro-level evacuation modeling analysis system which is named evacuation travel demand forecasting system. Franzese and Han(2001) developed a computer-based incident management decision aid system. Zou and Yeh.etc (2005)proposed a simulation-based emergency evacuation system for ocean city Maryland under hurricane attacks. U.Petruccelli (2003)research the knowledge under earthquake evacuation. Yuan Yuan et al. (2008) established path select model for emergency evacuation.
Under the internal concrete condition of collective transition and the network from multi-gathering points to multi-settling points when severe disasters struck, this paper discusses the distribution of multi-gathering points in the hazardous area and multi-settling points in the secure area, the respective suffers density in the corresponding gathering points, the respective accommodation of the corresponding setting points and the number of government vehicles for evacuation according to the computer simulation, and analyses the spatial point matching, path selecting and vehicle allocating methods during the evacuation model construction process. This paper takes Shandong province in China as the experimental area, based on WEBGIS and GOOGLE MAP API with computer programming language, to build the earthquake emergency evacuation platform.

\section{CAPACITY OF ROAD EVALUATING}

There are two goals need achieved when we evacuate persons from the disaster area to the safe area on the situation of quakes, that is to say, efficiency and safety. The efficiency indicates transition all victims from gathering points to setting points as soon as possible, relate to the road grades, the speed limits, the road length and the traffic flow and so forth. The safety denotes in the evacuation process we should ensure the victims safe because when passing through a part of disaster zone that building collapses, road surface damages and landslides were caused by the quakes. The strongest transportation capacity is equivalent to the lowest impedance on the contrary. Integrating it with the spring bounce model in physics: $\mathrm{F}=\mathrm{k} \times \Delta \mathrm{x}$, we propose one methods for structural objective function evaluation on road capacity, with the impedance as the criterion.

\subsection{Objective function evaluation on road capacity}

With the road impedance as the evaluation criterion, when it is a constant, the longer the road is, the stronger the impedance is, likewise, the two roads of the same length but different values of impedance, whose impedance is stronger 
with the longer length. The impedance is determined by two key factors: one is the transportation speed impedance determined by the road class, the speed limits, the road width and the traffic flow and so on, the other is the disaster impedance resulting from the road collapses and countercheck. According to the fore-mentioned theory, we can get the objective function evaluation on road capacity as follows:

$$
\mathrm{P}=\operatorname{Min} \sum \alpha 1 \times T i+\alpha 2 \times D i
$$

Ti denotes the efficient transportation time of some path. $\mathrm{Di}$ denotes the damage degree of some path surface. $\alpha 1$ and $\alpha 2$ denote the coefficients in the object function which is decided by experts' experience and actual road condition. To make it easy, we assume $\alpha 1=1, \alpha 2=1$ in the after-mentioned experiment, that is, efficient transportation time and the weight of the damage degree of the relevant path are identical.

\subsection{Objective function solution}

According to the fore-mentioned theory we derive the object function in formula 1 , and then solve the unknown quantity to get the summation P. Based on the past research and analysis on the road network data, many computation methods for road impedance have been proposed (Wang W.Q. and Zhou W et al. 2004; Zhuang Y. and Lv Sh., 2005), but we pick the model of calibration over again on the BPR function, rising in the research on traffic capacity, which is the key project in the ninth five-year plan, to formulate as below:

$$
\begin{aligned}
& \mathrm{Ti}=\frac{\mathrm{ti}}{\alpha 1} \times\left[1+\frac{Q i^{\beta}}{C i}\right] \\
& \alpha 1=\mathrm{V} 0 \times \frac{[1+Q i / C i]}{V d} \\
& \beta=\alpha 2+\alpha 3 \times\left(\frac{Q i^{3}}{C i}\right)
\end{aligned}
$$

$\mathrm{Qi}(\mathrm{pcu} / \mathrm{h})$ denotes the road flow in the real time. $\mathrm{Ci}(\mathrm{pcu} / \mathrm{h})$ denotes the road capacity. ti denotes the running time according to the designing speed. V0 $(\mathrm{km} / \mathrm{h})$ denotes the average speed. $V d(\mathrm{~km} / \mathrm{h})$ denotes the designing speed depending on the road ranking. $\alpha 1, \alpha 2 、 \alpha 3 、 \beta$ denotes regression parameters and correction factor respectively, with the actual values in the literature (Wang W. and Deng W., et al. 2001).

The disaster impedance often has a close correlation with the disaster type, the geographical conditions and economic situation in the location. It's the letter's key point that evacuation on the condition of earthquake, so we use the following formulation which proposed by Xu Tianben and Lv Jianghui (2002) to compute the disaster impedance.

$$
\mathrm{Di}=\mathrm{Pbi} \times \mathrm{Si} \times \mathrm{Ei}
$$

Pbi denotes the damage probability of the road. Si denotes the impedance degree of the same road. Ei denotes the corresponding exposure level.

$$
\mathrm{Si}=\mathrm{Hi} / \mathrm{Di}
$$

Hi denotes the average height of buildings in the road. Di denotes the valid width of the correspondent road.

$$
\mathrm{Ei}=\mathrm{ki} / \mathrm{Li}
$$

ki denotes the building density in the road. Li denotes the length of the road.

\section{MODELING MULTI-HIERARCHY EMERGENCY EVACUATION}

Modeling multi-hierarchy emergency evacuation is based on the road network data in the actual time, according to the road capacity evaluation criterion under the disaster, deriving from the fore-mentioned research, we can gain the support of the real-time and handled road network data, and then construct the multi-hierarchy evacuation model. We establish it according to the three levels.

\subsection{Spatial points matching}

The central mission of the model is to evacuate the people from the multi-gathering points in the disaster area to the multi-settling points in the secure area when disaster is striking. How to finish the evacuation from a certain gathering point to some setting points needs the points matching on the basis of the spatial property between the gathering point and setting point. This paper supposes that the epicenter locates a certain center of the experimental area, then designates the dangerous region, buffer zone and safe region by simulating quake magnitude, and picks multi-gathering points and multi-settling points in the corresponding zones. The points must satisfy certain criteria. The gathering points ought to be in the disaster region which is convenient to gathering people, where is no tall building, which is far away from the disaster origin and which is near to the traffic hub zone. The setting points ought to be in the safe region away from the dangerous area. We usually take hospitals, schools and squares as setting points in consideration of factors such as the relief materials conveyance, the real-time cure to victims and adopt ability for evacuation persons. As long as the points are fixed, we can match the multi-gathering points and the multi-settling points by spatial analysis.

(1) Spatial analysis and Voronoi diagram

Spatial analysis is an analysis technology of spatial data based on the location and morphological feature of geographic object, with the goal of learning about the events in space, extracting and conveying spatial information. Spatial analysis includes spatial location, spatial distribution, spatial morphology, spatial distance, spatial orientation, topology, similarity and relevance amounting to five aspects. In this paper we mainly rely on the spatial location, spatial distribution, spatial orientation and topology of multi-gathering points and multi-settling points, and settle the matching problem according to the dissection trait of Voronoi diagram.

The Voronoi diagram (Franz A. and Rolf K., 1996) is correlative with discrete points, divided the plane into several region with only one point in it, which is the nearest assemble points to the only point. It is used for spatial dissection whose 
leading character is that the distance between the internal point of the polygon composing by some points in the plane and its generator is nearest. We use weighted Voronoi diagram as spatial dissection concerning the population and distribution density in the gathering points and the admit population in the setting points.

There are many ways to generate Voronoi diagram (Zhang W. and $\mathrm{Gu}$ Ch. L., 2000; Zhen Ch. H.et al. 2011). The traditional one is provided that $\mathrm{n}$ discrete points $\mathrm{P}=\{\mathrm{p} 1, \mathrm{p} 2$, $\mathrm{p} 3 \ldots . . . \mathrm{pn}\}$ in the plane, so the relevant Voronoi diagram $\mathrm{Ti}=\{\mathrm{x} \mid \mathrm{d}(\mathrm{x}, \mathrm{pi})<\mathrm{d}(\mathrm{x}, \mathrm{pj}) / \mathrm{pi}, \mathrm{pi} \neq \mathrm{pj}\} . \mathrm{d}$ denotes Euclidean distance. The weighted one is a little different from it. Likewise, providing that $\mathrm{n}$ discrete points $\mathrm{P}=\{\mathrm{p} 1, \mathrm{p} 2, \mathrm{p} 3 \ldots \ldots \mathrm{pn}\}$ in the plane, and $\mathrm{n}$ positive real numbers $\lambda=\{\lambda 1 、 \lambda 2 、 \lambda 3 \ldots \ldots\}, \mathrm{Vn}=\mathrm{n}(\mathrm{Pi}, \lambda \mathrm{i})=\mathrm{\cap}\{\mathrm{P} \mid \mathrm{d}(\mathrm{p}, \mathrm{pi}) / \lambda \mathrm{i}<$ $d(p, p i) / \lambda j, i \neq j\}$, divided the plane into $n$ parts, $\lambda i$ denotes the weight of the point.

(2) Multi-gathering points and multi-settling points space matching

According to the coordinates of the simulating points and the road network structure, we construct the weighted Voronoi diagram and road network classified layer, and then make them stack. We can analyses the spatial point matching in the light of the overlap layer. As shown in the following fig.1, we can see the polygon proximity relations in the radioactive region constructed by every point. In accordance with road capacity in the zone where there is a point, the population in every gathering point and the admit population in every setting point, selecting some setting points to every gathering point.

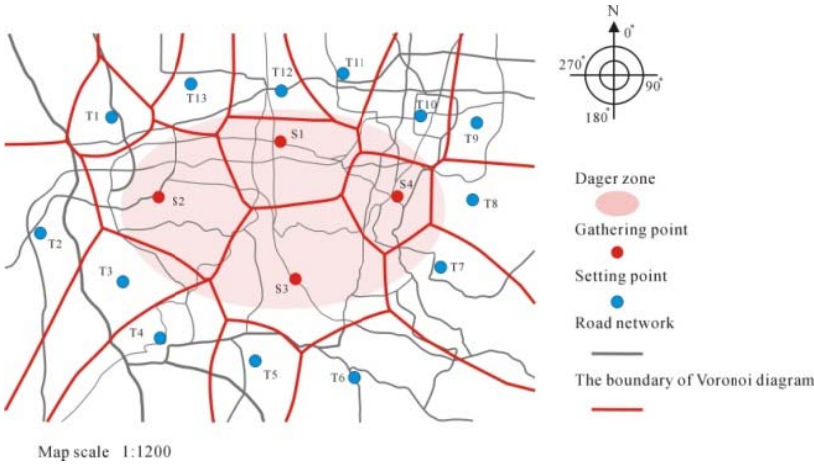

Figure 1. Overlaying layers

According to the road network layer which describes the road evacuation capacity as a group of lines in different thickness and the weighted Voronoi diagram which is established by the spatial location of multi-gathering points and multi-settling points, we can obtain the following table:

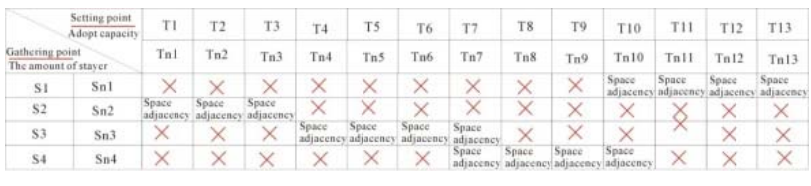

Table 1. Space adjacent table

Tab. 1 is the space adjacency table about the space simulation relationship of the multi-gathering points and multi-settling points, we can see from the table that the setting points S1 has four adjacent points here: T10, T11, T12, T13; S2 has three adjacent points here: $\mathrm{T} 1, \quad \mathrm{~T} 2, \mathrm{~T} 3 ; \mathrm{S} 3$ has four adjacent points here: T4, T5, T6, T7; S4 has four adjacent points here: T7, T8, T9, T10.According to the topological relationships of the various gathering points and the weights about the ability of the road traffic, we can get the Tab. 2 as below.

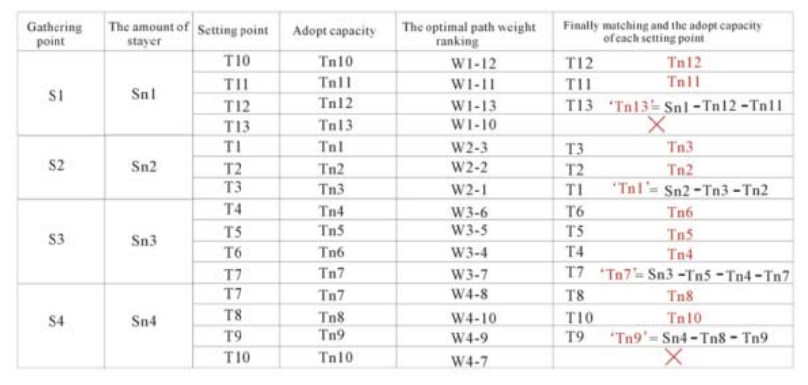

Table 2. Spatial point matching table

Tab.2 is ultimate space-point matching program which is based on the topological relationships of the two kind of points , and the traffic capacity of road network and the accommodation of population of each point. From the table we can see that, according to the simulated population of $\mathrm{S} 1$ and the adopt capacity of each setting points: T10, T11, T12, T13 which should consider firstly, we can define the matching setting point of S1 are: T11, T12, T13, Because we should distribute the population to the best place, when the population acceptance of point T12, T11 is full, the free population acceptance of $\mathrm{T} 13$ is the difference number between the original population acceptance of $\mathrm{T} 13$ and the remaining population after the transmission between $\mathrm{S} 1$ to T12, T11 has been completed. According to the method we can define the corresponding setting points of each gathering point and can get the number of the population distribution of the setting points.

\subsection{Path searching}

To transfer the disaster victims from gathering points to setting points, we have several routes to choose from. And how to select the optimal path to make the disaster victims from the rally points to settle safely and efficiently is the most important part in constructing emergency evacuation model under disaster condition. According to the spatial point matching way obtained based on the weighted Voronoi figure and layers overlay of road network layer, we get some information of several setting points that is considered preferentially in each gathering point, and that is the path searching of single Origin to Multi-destinations. Usually it's impractical if only choose one path as evacuation route from a rally point to another site, because one path often causes the short time congestion, adding to the road transport pressure, and directly cause the delay of overall evacuation time. We use the K optimal paths based on maximum flow on the premise of global optimum (Kou W. H. et al.2009; Ch J. Ch. et al. 2007; Ahuja R K and Magnanti T L, 2000), selecting the optimum several paths from single gathering point to single setting point according to the evaluation criteria of road transportation ability, namely, the optimal $\mathrm{K}$ path. The basic thought of the optimal $\mathrm{K}$ path selection method is that there are several roads from a gathering point to other setting points, and each road is made by some sections. According to the discretion of the impedance of the sections, we determine the overall impedance of each understanding path from gathering points to setting points, then we select the path of least resistance as optimal path, which in 
turn to the second best selection, the optimal path selection again until K optimal path.

3.2.1 Multi-paths problem under the maximum flow constraints: The assessment of traffic capacity of selected section is based on the information of real-time dynamic traffic flow, road damage degree and static road conditions. In order to realize the Real-time optimal quality of selected road during the process of evacuation under disaster, we adopt the $\mathrm{K}$ optimal path method based on the maximum flow to solve the problem of increasing traffic flow and affecting overall evacuation speed caused by short term personnel congestion. We recode the population distribution information of each section in the links node, as below.

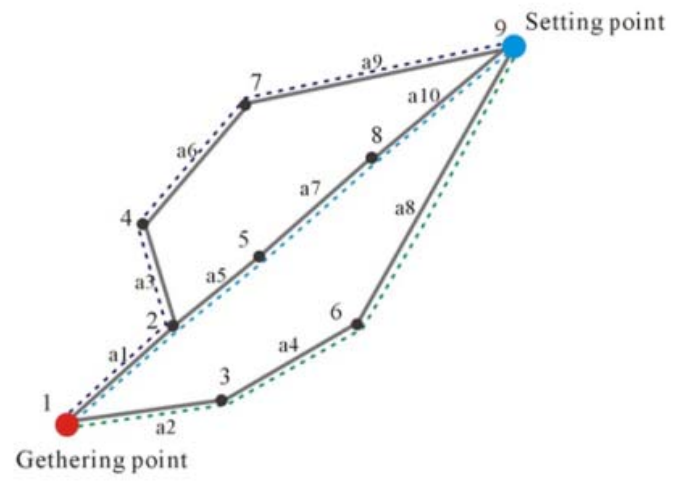

Figure 2. Population distribution based on max-flow

The sections weight in figure is assessed by the reciprocal of the impedance of those roads which is to be assessed the transportation capacity, the a1, a2, a3......separately stands for the weight of each section. $n_{1-2}$ indicates the distributed population on the section from link node 1 to node 2 .

Based on the above figure, starting there are three paths available to evacuate people from the gathering point to setting point. Three paths, respectively, go through different nodes: $\{1$, $2,4,7,9\} ;\{1,2,5,8,9\} ;\{1,3,6,9\}$, road sections with link nodes is already available in data of road network. According to relationship among road sections, nodes and the high flow of people in the figure, the following table is obtained:

\begin{tabular}{|l|l|l|l|l|l|}
\hline Road sections & $1-2$ & $1-3$ & $2-4$ & $2-5$ & $\cdots$ \\
\hline $\begin{array}{l}\text { Road transportation } \\
\text { ability }\end{array}$ & $\mathrm{a} 1$ & $\mathrm{a} 2$ & $\mathrm{a} 3$ & $\mathrm{a} 4$ & $\cdots$ \\
\hline $\begin{array}{l}\text { Distributed } \\
\text { population }\end{array}$ & $n_{1-2}$ & $n_{1-3}$ & $n_{2-4}$ & $n_{2-5}$ & $\cdots$ \\
\hline
\end{tabular}

Table 3. Information table

Based on the ideas of maximum flow, we adopt the following formula to calculate the final number of distribution people of each path:

Calculation of the population flow between 1-2 nodes: $n_{1-2}=N \times \frac{a 1}{(a 1+a 2)}$; the population flow between 1-3 nodes: $n_{1-3}=N \times \frac{a 2}{(a 3+a 5)}$; the population flow between 2-4 nodes: $n_{2-4}=n_{1-2} \times \frac{a 3}{(a 3+a 5)}$; the population flow between 2-5 nodes: $n_{2-5}=n_{1-2} \times \frac{a 5}{(a 3+a 5)} ;$ then according to the calculation law, the population on each road section is obtained.
After the population on each section is figured out, population needs to be identified from an assembly point to a certain settlement point on a certain accessible road transport. During the assignments process based on the maximum flow, we will record the flow of people assigned to road sections in the node information. In order to determine the final person flow on a path, you should compare the population flow recorded in all the nodes included in the path, then take minimum value as the final population flow on the entire path.

3.2.2 Road searching in network hierarchy: It will has low efficiency when path searching processes based on max-flow theory to calculate multiple optimal paths which face to large area veal time and including POI(point of interesting) information road network data. Traditional path searching algorithms could find the optimal path from start point to destination point while those algorithms couldn't figure out well if the question is very complicated. Dijkstra algorithm is a typical shortest path algorithm to calculate a node to all other nodes of the shortest path. Because Dijkstra algorithm is a step by step search algorithm for each vertex $n$ reservations so far found from them to $m$ shortest path to work, it can arrive at the optimal solution, but because of its calculation of the node traversal, so inefficient. That is to say, Dijkstra shortest path searching algorithm suites to small data sets rather than large data sets. To solve this problem, some optimal route algorithms are proposed (Xiang J. P. et al.2011; Qiao L. et al. 2011).As to various networks, the efficiencies of all optimal route planning algorithms are different. And there is no algorithm that has the highest efficiency to all kinds of networks. Based on the special road network and the detailed requirements of emergency evacuation model under the disaster condition, the road network hierarchy searching is used to establish mathematics model for path optimizing searching. The whole algorithmic principles are shown in the figure below.

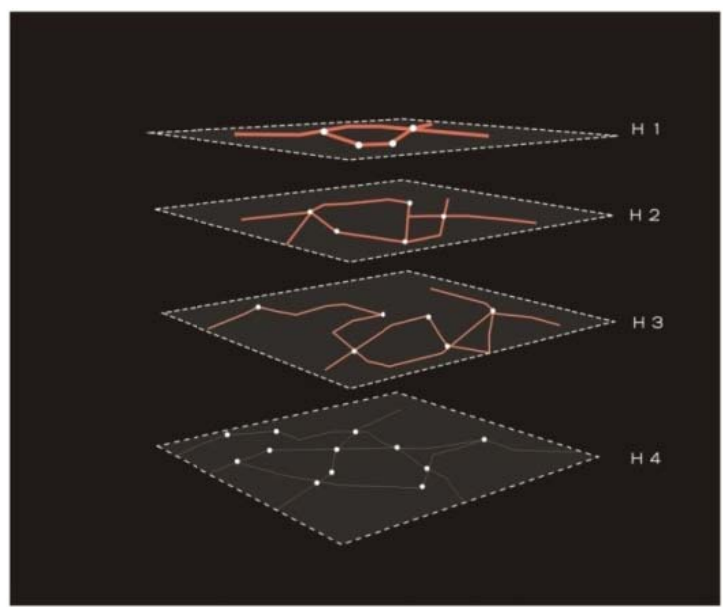

Figure 3 .Road network layers

All road network data is divided into four specific layers by the ability of road evacuation which is represented as : $\mathrm{H}=\{\mathrm{H} 1, \mathrm{H} 2, \mathrm{H} 3, \mathrm{H} 4\}$. The graph of road network $\mathrm{G}=\{\mathrm{V}, \mathrm{E}, \mathrm{W}\}$ and $\mathrm{V}$ means the set of road nodes, $\mathrm{E}$ means the set of sections of road, $\mathrm{W}$ means the set of weight of each section of road, such as: $\quad V i=(v 1, v 2, v 3 \ldots \ldots) ; E i=(\mathrm{e} 1, \mathrm{e} 2, \mathrm{e} 3 \ldots \ldots) ; \mathrm{Wi}=$ $(w 1, w 2, w 3 \ldots . .$.$) .The threshold value of each layer is defined$ based on the ability of road evacuation. Assume that the threshold value: $\quad \zeta=\{\zeta 1, \zeta 2, \zeta 3, \zeta 4\}$ $\zeta 1=\left\{\zeta 1_{\min }, \zeta 2_{\max }\right\} ; \zeta 2=\left\{\zeta 2_{\min }, \zeta 2_{\max }\right\} ; \zeta 3=$ 
$\left\{\zeta 3_{\min }, \zeta 3_{\max }\right\} ; \zeta 4=\left\{\zeta 4_{\min } ; \zeta 4_{\max }\right\}$. For each layer Hie H, we compare $\mathrm{Wi}(\mathrm{v}$, e) with threshold $\zeta \mathrm{i}$ and make sure the current road data belongs to which layer. After all road data has compared with threshold, we will get four sets such as: $\mathrm{Hi}=\{\mathrm{Vi}$, Ei, Wi $\}, i=1,2,3,4$. Searching the $\mathrm{K}$ optimal paths from gathering point to setting point based on following steps:

Step1: Finding the nearest road to the current gathering point and compare the ability of road evacuation with threshold of each layer;

Step2: Looking for the other collinear points with current gathering point and compare those points with threshold value to make sure they are located in which layer. Assume that the set of road nodes of collinear road section be represented as: $\mathrm{N}=\{\mathrm{n} \mid \mathrm{ni} \in \mathrm{Ls}, \mathrm{i}=1,2 \ldots \ldots\}$.

Step3: Taking one node from the set $\mathrm{N}$ to begin the next step of path searching.

Step4: If the current road node is located in the highest layer then search the next section of road which is collinear with it. The end node of the current section of road as the current node, that is, to reuse the same method over and over again until the current node is collinear with settling point.

Step5: If we can't find the collinear node with current node in the current data layer, we need to find the mapping point of current node in the next layer and reuse Step 4 and Step 5 until to find all the successful way from all gathering points to all setting points. The concrete methods are shown in the figure below.

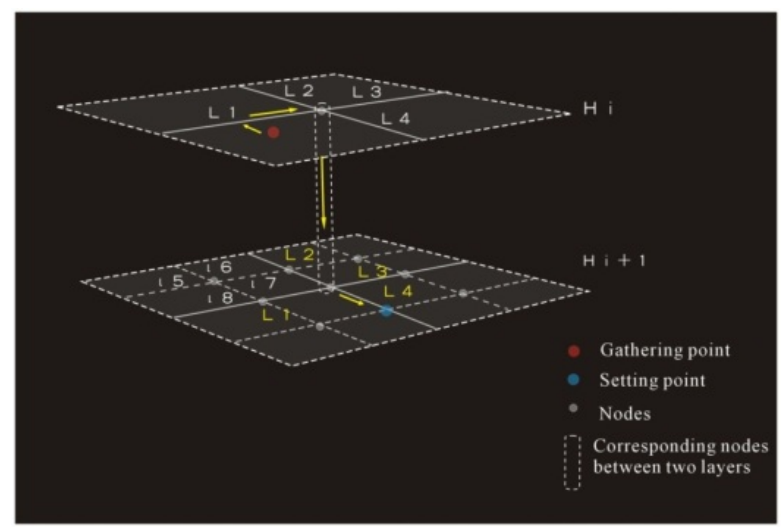

Figure 4. Detailed network

\subsection{Vehicle Assignment}

The traffic flow's assignment has a strong influence on the unblocked transportation of one road. When constructing the emergency evacuation platform, we put traffic flow's assignment into vehicle's assignment, considering the special situation of collective evacuation and special strategies of specific road section after the disaster happened. By searching paths and allocating node flow, we can get the population on each single road of the $\mathrm{K}$ optimal paths from certain gathering point (Origin) to certain setting points (destination). Supposed starting from gathering points to setting points, the number of population allocated to each evacuation path are $\mathrm{Q}_{1}, \mathrm{Q}_{2}$, $\mathrm{Q}_{3} \ldots \mathrm{Q}_{\mathrm{m}}$. According to the computational formula of evacuation time, we can acquire the single evacuation time $\mathrm{ET}_{\mathrm{i}}$ (the single evacuation time of the path numbered i) of each path. Assumed that there are $m$ optimal evacuation paths, the single evacuation time of each path can be expressed as $\mathrm{ET}_{1}, \mathrm{ET}_{2}, \mathrm{ET}_{3} \ldots \mathrm{ET}_{\mathrm{m}}$. Objective function of vehicle assignment was established aiming at seeking overall optimal.
3.3.1 Establishing objective function of vehicle assignment: While the evacuation time of single path is approximate to mean value of the finished evacuation time of all the emergency evacuation paths, we can treat it as overall optimal, that's when the value of the objective function $\mathrm{F}$ above is infinite close to zero. The amount of vehicles for each optimal path is the best result. In practical application, we obtain the number of vehicles allocated for each path by setting $\mathrm{F}$ as zero.

$$
\mathrm{F}=\sum_{\mathrm{i}=0}^{\mathrm{m}}\left(\frac{\mathrm{ETi} \times \mathrm{Qi}}{\mathrm{Ni} \times \mathrm{a}}-\sum_{\mathrm{i}=1}^{\mathrm{m}} \frac{\mathrm{ETi} \times \mathrm{Qi}}{\mathrm{Ni} \times \mathrm{m} \times \mathrm{a}}\right)^{2}
$$

3.3.2 Solving objective function: Supposed there are $m$ paths from all the gathering points to all the setting points, the single evacuation time of each path in all the $m$ paths is $\mathrm{ET}_{\mathrm{i}}$. Assumed that the number of people certain vehicle can carry is a, so the number of vehicles allocated for each path is $\mathrm{N}_{\mathrm{m}}$, the total amount of the vehicles is $N . n_{i}$ means there are $n$ road sections on evacuation path numbered i. $t_{i j}$ indicates the single evacuation time of road section $j$ on path $i$.

$$
\left(\begin{array}{c}
\mathrm{N}=\mathrm{N} 1+\mathrm{N} 2+\ldots \mathrm{Nm} \\
\frac{\mathrm{ETi} \times \mathrm{Qi}}{\mathrm{Ni} \times \mathrm{a}}-\sum_{\mathrm{i}=1}^{\mathrm{m}} \frac{\mathrm{ETi} \times \mathrm{Qi}}{\mathrm{Ni \times m \times a}}=0 \\
\sum_{j=0}^{\mathrm{ni}} \mathrm{tij}=\mathrm{ETi}
\end{array}\right)
$$

\section{EXPERIMENT}

We chose Dezhou city, Shangdong province as the experimental area to set up emergency evacuation model platform. Assume that a point in Dezhou city as the center of the earthquake. Setting up emergency evacuation model platform under the condition of earthquake.

\subsection{Establishing real-time database}

Flexible emergency evacuation model can provide scientific and reasonable evacuation strategy for policy makers in accordance with the categories, severity and area of disasters and also need real-time dynamic so as to adapt to constant disasters while some disasters happen. To make the latest data be used by platform, we chose to use real-time database system (Y.Kim and S.H.Son, 1996; Jan.L, 2008.) Usually, real-time information only guarantees to realize relative real-time dynamic in the shortest period as soon as possible. Real-time database mainly stores the real-time road network data and disaster data. When platform uses these data, it needs to be pretreated. Data pretreatment includes road network traffic capacity monitoring in a period, disaster degree monitoring, network topology construction and experimental gathering dot and setting dot selection. Real-time database framework is as follows: 


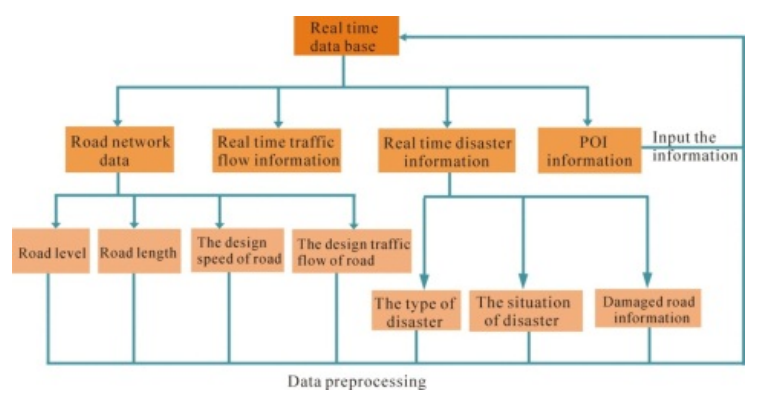

Figure 5. The conceptual graphs of Real-time data base

\subsection{Road capacity assessment of the experimental zone}

Road capacity assessment is determined by real time road data and the type of disaster of the experimental zone. From literature ${ }^{[11]}$ we can get each parameter and according to the objective function of road network capacity assessment which has explained in the earlier paragraph to get the capacity of each section of road. Assume that the road network of whole experimental zone as a undirected graph: $\mathrm{G}=\{\mathrm{V}, \mathrm{E}, \mathrm{W}\}, \mathrm{V}=\{\mathrm{v} 1$, $\mathrm{v} 2, \quad \mathrm{v} 3 \ldots \ldots\}, \quad \mathrm{E}=\{\mathrm{e} 1, \mathrm{e} 2, \mathrm{e} 3 \ldots \ldots\}, \quad \mathrm{W}=\{\mathrm{w} 1, \quad \mathrm{w} 2$, $\mathrm{w} 3 \ldots \ldots . . .$. . And we store the value of road capacity to set $\mathrm{W}$ and correspond with the set $\mathrm{V}$ and $\mathrm{E}$.

We can get the topological information table (tab.4 and tab.5) of road data after finish the building of topological of road network of the experimental zone.

\begin{tabular}{|c|c|c|}
\hline Node ID & XCoord & YCoord \\
\hline 0 & 116.892997 & 37.207258 \\
\hline 1 & 116.231477 & 37.478929 \\
\hline$\ldots \ldots$. & $\ldots \ldots \ldots \ldots$ & $\ldots \ldots \ldots$ \\
\hline
\end{tabular}

Table 4. Node table

\begin{tabular}{|c|c|c|c|c|c|}
\hline $\begin{array}{c}\text { Road } \\
\text { ID }\end{array}$ & $\begin{array}{c}\text { Start } \\
\text { Point }\end{array}$ & $\begin{array}{c}\text { End } \\
\text { Point }\end{array}$ & Length & $\begin{array}{c}\text { Transportation } \\
\text { Time }\end{array}$ & Weight \\
\hline 0 & 73 & 75 & 1.18 & 0.01986 & 0.01993 \\
\hline 1 & 72 & 73 & 0.71 & 0.01195 & 0.01202 \\
\hline$\cdots \cdots$ & $\cdots \cdots$ & $\cdots \cdots$ & $\cdots \cdots$ & $\cdots \cdots$ & $\cdots \cdots$ \\
\hline
\end{tabular}

Table 5. Road network table

The information of road sections is described by relational table. Start Point and End Point record the two point information of one road section. And we use ID number to record the road section. "Length" record the length information of each road section; "Transportation Time" record the evacuation time of each road section; "Weight" record the capacity of each road section under the disaster condition; the node information is recorded in the Node relational table. In this table we use ID number to record the nodes number and utilize "XCoord" and "YCoord "to record the coordinates for all nodes of the road network. According to the ID number in the Node table and the information of "Start Point" and "End Point" in the Road network relational table, we can connect two tables easily.
4.3 The multi-gathering points and multi-settling points selection and the internal mechanisms of points matching

The multi-gathering points and multi-settling points are determined by the real disaster situation of experimental zone. According to the real situation of Shang Dong province De Zhou city and to analog the situation of disaster, we choose four gathering points according to (Current J.M. and Schilling D., 1990; Fang L. and He J.M., 2003; Chang Y.L. and Wang W., 2005): S1, S2, S3, S4 and eleven setting points : T1, T2, T3, T4, T5, T6, T7, T8, T9, T10, T11. According to the above mentioned method, we can build the road network hierarchical diagram to overly on the weighted Voronoi diagram which is established by the special position of gathering points and setting points. The principle of two layer overlying is described as follows (fig.6). Then we need to store the gathering points and the setting points matching information to the real time data base.

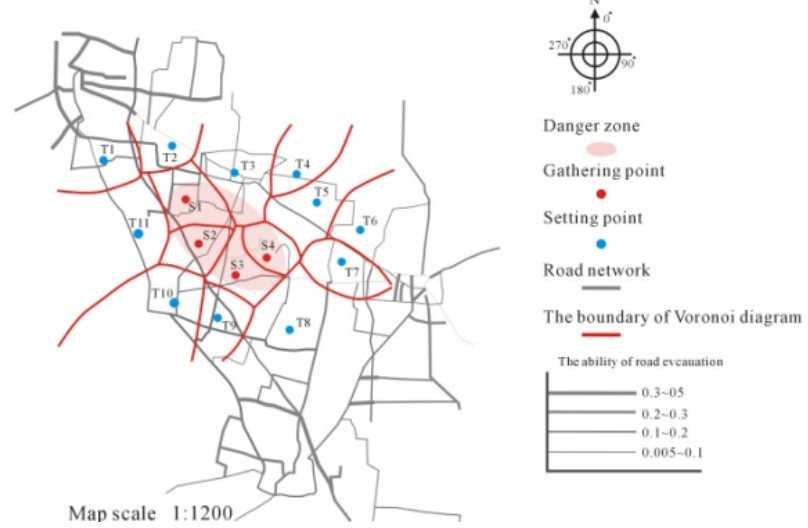

Figure 6. Real point matching diagram

\subsection{Experiment}

Based on the establishing method of emergency evacuation model in this paper and connected with the Web GIS technology and Google Map API technology, we use C\# program language to establish the emergency evacuation plot which be called as : traffic network emergency decision support system of cross area. Follow the function buttons which are provided by the traffic network emergency decision support system of cross area, we can get the position of all the gathering points and setting points, and input the real data which include amount of stayers in gathering points, the adopt capacity of setting points, the position of disaster happening and the range of disaster. Meanwhile we can set the relevant parameters such as the amount of vehicles to get the final evacuation scenario as follows (fig.7).We assume that the population of the area is 25000 , and the government has 1000 vehicles to evacuate. According to shown in the figures: we can get the following final distribution form:

\begin{tabular}{|l|l|l|l|l|}
\hline $\begin{array}{l}\text { Gathering } \\
\text { point }\end{array}$ & $\begin{array}{l}\text { Setting } \\
\text { point }\end{array}$ & $\begin{array}{l}\text { Adopt } \\
\text { capacity }\end{array}$ & $\begin{array}{l}\text { Vehicle } \\
\text { assignmen } \\
\text { t }\end{array}$ & $\begin{array}{l}\text { Suppl- } \\
\text { ement }\end{array}$ \\
\hline \multirow{3}{*}{ ID:5/500 } & ID:224/200 & 200 & 4 & \\
\cline { 2 - 5 } & ID:225/150 & 300 & 3 & \\
\cline { 2 - 5 } & ID: $146 / 150$ & 150 & 3 & \\
\hline
\end{tabular}

Table 6.Scheme table 


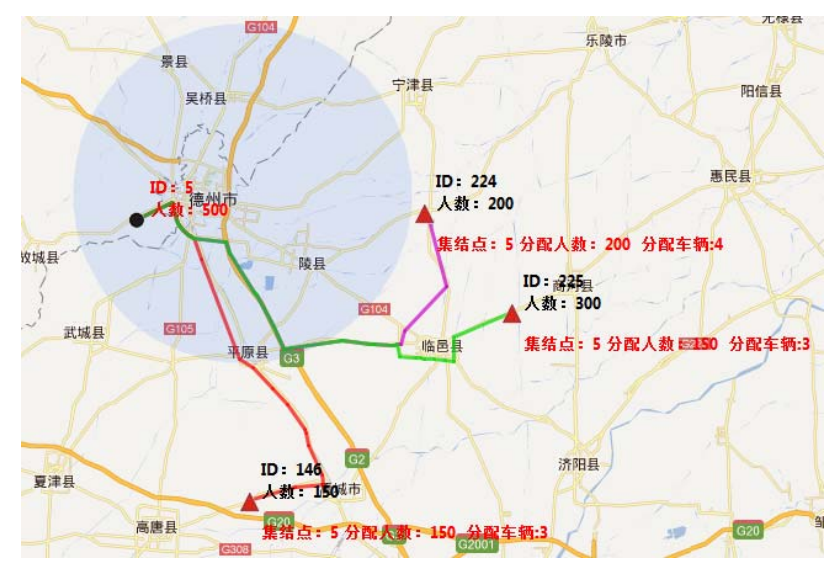

Figure 7. The route planning and vehicles assignment Result

\section{SUMMERY}

Frequent occurrences of natural disasters in recent years have caused concern about studying the emergency evacuation model. Based on examining the system of emergency evacuation and predicting the happening of disaster, to minimize damage and avoid loss of life is of great importance for safeguarding and seeking social stability and promoting common development. In this paper we propose several new methods for building emergency evacuation model which include using weighted Voronoi diagram to get the matching of multi-gathering points and multi-settling points, applying hierarchical road data to optimize path searching method, establishing vehicle assignment model in the base of the global optimization. Those approaches are not only used for collective evacuation in china when an earthquake occurs but also be adopted for other disaster in through updates the real time data base. And how to develop and optimize those methods to promote the further development of emergency evacuation model is still a challenge.

\section{ACKNOWLEDGMENTS}

This work is supported by funding from Major Project of Chinese National Programs for Fundamental Research and Development (973 program No. 2012CB725300), and the Fundamental Research Funds for the Central Universities, and the projects funding of LIESMARS. The authors also gratefully acknowledge the helpful comments and suggestions of the reviewers, which have improved the presentation.

\section{REFERENCE}

Antoine G, Hobeika, Changkyun K., 1998. Comparsion of traffic assignments in evacuation modeling, IEEE Transactions on Engineering Management, 45(2), pp.192-198.

Ahuja R K, Magnanti T L, 2000.Network flows: theory, algorithms and applications. New Jersey: Pren-tice-Hall, pp.34-78.

Cova T J., Johnson J P., 2003. A network flow model for lane based evacuation routing. Transportation Research Part A, 37, pp. 579 - 604.

Ch J. Ch., Cheng R.G., Hao T. Sh., Yih-Shen Chen., 2007. Maximum freedom last scheduling algorithm for downlinks of DSRC Networks .IEEE Intelligent Transportation Systems Society, 8 (2), pp.223-232 .

Current J, M in H, Schilling D., 1990. Multiobjective analysis of facility location decisions. European Journal of Operational Research, 49(3), pp.295-307.

Chang Y.L., Wang W., 2005. Optimized location model and algorithm of urban emergency systems. Journal of management sciences in china, 8(1), pp:13-16.

Fang L., He J.M., 2003. Models and Algorithms of optimized location of the emergency system. Systems engineering, $18(1)$, pp.49-54.

Franzese.O and L.Han, 2001, Traffic modeling framework for hurricane evacuation, Technical paper No.01-2591.80th annual meeting of the transportation research board, Washington, D.C.

Franz.A., $\quad$ Rolf.K., 1996.Voronoi diagrams.http://www.pi6.fernuni-hagen.de/publ/tr198.pdf, pp.2-11.

Jernigan Post, Buckley and Schuh, 2000.Evacuation travel demand forecasting system: technical memorandum2.Inc. Tallahassee, Florida.

Jan.L, 2008.Realtime data base systems.http://www.cs.helsinki.fi/u/jplindst/papers/rtds.pdf, pp.6-24.

Kou W. H., Dong X., Lv L. J., 2009. An algorithm for the minimum cost max-flow with limited flow between two notes in the traffic and transportation network . Journal of Lanzhou Jiaotong University, 28(6), pp.105-107.

Lewis D C,1985. Transportation planning for hurricane evacuations. ITE Journal, 55 (8), pp. 31 - 35.

Ma L. L., Zhang Y. H., 2011. Property of divisional weighted voronoi diagram and area calculation. Computer science, 38(2), pp.195-198.

N.Zou, S.T.Yeh and G.L. Chang, 2005, A simulation-based emergency evacuation system for ocean city Maryland under hurricane attacks, The 84th Annual meeting of the transportation rsearch board, Washington, D.C.

Qiao L., Jing H., Li Y.X., Huang J. Y., 2011.Mix K-means clustering with ant colony optimization used in city emergency erescue decision-making. China public security(academy edition), 23(2), pp.53-57.

U.Petruccelli, 2003, Urban evacuation in seismic emergency conditions, ITE journal, vol.73(8), pp.25-30.

Wang W.Q., Zhou W., L L.S., 2004.Theory and application of path impedance function. Highway and transportation research, 21(9), pp.82-85.

Wang W., Deng W., Yang Q., etc. 2001.Construction and Management of road network planning. Peiking: science press, pp.101-103.

Xu T.B., Lv J.H., 2002. Application and establishment of mode of the minimum risk path selection for earthquake relief. Journal of Engineering of National Taiwan University, 85, pp.32-47.

Xiang J. P., Wang Y., Hu J., 2011.Constrained optimized path searching algorithm based on road network. Computer engineering, 37(12), pp.53-55.

Yuan Y., Wang D.W., Jiang Zh., Sheng Y., 2008. Multi-objective path selection model for emergency evacuation taking into cccount the path complexity. Openatons restarch and manacement science, 17(5), pp.74-77.

Y.Kim, S.H.Son, 1996.Supporting predictability in real-time database systems, IEEE Real-Time Technology and Application Symposium(RTAS'96), Boston, MA, June

Zhuang Y., Lv Sh., 2005. Study on the link traveling time function for urban road based on TransCAD.Communications standardization, 10, pp.122-124.

Zhang W., Gu Ch. L., 2000. Model system for urban and regional planning. Nanjing: Southeast University Press, 
ISPRS Annals of the Photogrammetry, Remote Sensing and Spatial Information Sciences, Volume I-2, 2012 XXII ISPRS Congress, 25 August - 01 September 2012, Melbourne, Australia

pp.118.

Zhen Ch. H., 1999. Research of economic space organization in

Henan Province. Kaifeng: He Nan University, Master's thesis, pp.38-45. 